\title{
Leader-follower Formation Control of Mobile Robots with Sliding Mode
}

\author{
Wenhao Zheng, Yingmin Jia \\ The Seventh Research Division and the Center for Information and Control, \\ School of Automation Science and Electrical Engineering, \\ Beihang University (BUAA), 37 Xueyuan Road, Haidian District, \\ Beijing 100191, China \\ E-mail: zhengwenhao152@163.com,ymjia@buaa.edu.cn \\ www.buaa.edu.cn
}

\begin{abstract}
This paper considers the formation control of nonholonomic mobile robots. The formation problem is converted to the error model based on the leader-follower structure. A sliding mode controller, which is proved to be globally finite-time stable by Lyapunov stability theory, is presented in this study. In addition, a continuous reaching law is designed to reduce the chattering which caused by the computation time delays and limitations of control. Simulation results verify the feasibility and effectiveness of the control strategy.
\end{abstract}

Keywords: nonholonomic mobile robot, leader-follower, formation control, sliding mode, continuous reaching law.

\section{Introduction}

Formation control of differential-drive mobile robots have been widely researched in recent years. The mobile robots are supposed to maintain the desired geometric configuration in lots of different situations including personnel rescue, logistics transportation, military affairs and environmental exploration. Various formation control strategies have been proposed, such as feedback linearization ${ }^{1}$, backstepping ${ }^{2}$, sliding-mode control $^{3}$ and sorts of intelligent control method. In Ref.3, the kinematic model and the dynamic model were both considered, then a sliding mode controller was proposed to deal with model uncertainties and disturbances, furthermore, the voltage inputs of driving motors were taken as control inputs, which are more realistic than the velocity. Basic controller based on backstepping method was presented in Ref.4, a bioinspired neurodynamics based approach was further developed to solve the impractical velocity jumps problem. In Ref.5, a controller with time-varying parameters which were designed to limited control inputs, was presented by Lyapunov theory, and geometric analysis contributed to the designment of optimal feedback functions which made the controller more effective.

This paper mainly focuses on the formation control of nonholonomic mobile robots with sliding mode. The formation problem is proposed with leader-follower setup, which is used to deduce the error model. Then, a simple sliding mode surface is chose to design control law. And a continuous reaching law is presented to reduce the chattering. Moreover, the formation error model is proved to be globally finite-time stable. Comparing with existing results, this paper primarily contributes to the novel solution of the formation control with sliding mode.

The structure of the rest paper is organized as follows. The section 2 introduces leader-follower system and the formation error model. In the section 3, the sliding mode controller is designed with a continuous reaching law. Simulation results are 
presented to show the validity of the control law in the section 4. In the end, the section 5 summarizes the whole paper and draws the conclusion.

\section{Problem Statement}

\subsection{Kinematic model of nonholonomic mobile robot}

In this paper, the differential-drive mobile robot is considered as the research object, which is subject to nonholonomic constraint (1).

As shown in figure 1 , let $v_{L}$ and $v_{R}$ be the velocities of the left and right driving wheel. The control inputs are usually taken as the linear speed $v$ and the rotational angular velocity $\omega$. The relation

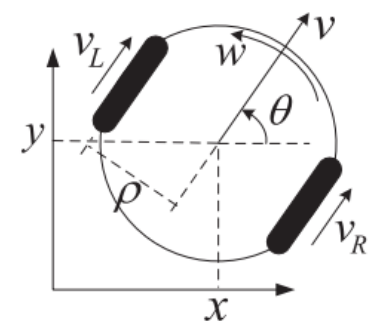

Fig. 1. The configuration of a two-wheeled mobile robot.

between control inputs and driving wheels can be expressed as (2). In practical systems, control inputs of mobile robots are bounded.

$$
\begin{gathered}
\dot{y} \cos \theta-\dot{x} \sin \theta=0 \\
v=\left(v_{L}+v_{R}\right) / 2, \omega=\left(v_{R}-v_{L}\right) / 2 \rho \\
\dot{x}=v \cos \theta, \quad \dot{y}=v \sin \dot{\theta}, \quad \dot{\theta}=\omega
\end{gathered}
$$

where $(x, y)$ are the robot position and $\theta$ is the robot orientation. Moreover, the kinematic of the mobile robot can be expressed as equation (3).

\subsection{Leader-follower system}

In the figure 2, leader-follower formation structure is presented in Cartesian coordinates. The nonholonomic mobile robot $R_{l}$ is treated as the leader. The center of the leader $C_{l}\left(x_{l}, y_{l}, \theta_{l}\right)$ describes the position and the orientation of the leader in the world frame. And $\left(v_{l}, \omega_{l}\right)$ are control inputs. The follower is the robot $R_{f}$. The center of the robot $C_{f}\left(x_{f}, y_{f}, \theta_{f}\right)$ and inputs $\left(v_{f}, \omega_{f}\right)$ have the analogous definitions like the leader. Assume that the distance between the point $O_{f}\left(x_{o f}, y_{o f}, \theta_{f}\right)$ and $C_{l}$ is $d$. The relation can be presented as (4)

$$
\begin{aligned}
& x_{o f}=x_{f}+d \cos \theta_{f} \\
& y_{o f}=y_{f}+d \sin \theta_{f}
\end{aligned}
$$

Differentiating the above equations leads to

$$
\begin{aligned}
& \dot{x}_{o f}=v_{f} \cos \theta_{f}-d \omega_{f} \sin \theta_{f} \\
& \dot{y}_{o f}=v_{f} \sin \theta_{f}+d \omega_{f} \cos \theta_{f}
\end{aligned}
$$

The desired geometric shape can be denoted by

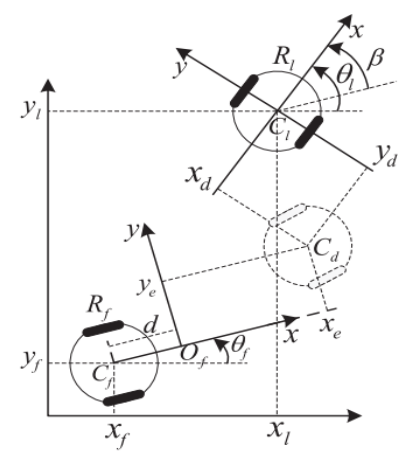

Fig. 2. Leader-follower formation structure

coordinate representation. The expected position of the follower should be expressed as $\left(x_{d}, y_{d}\right)$ in the coordinate system of the leader. And the real position $C_{d}\left(x_{c}, y_{c}\right)$ in world frame can be deduced as equation (6).

$$
\left(\begin{array}{l}
x_{c} \\
y_{c}
\end{array}\right)=\left(\begin{array}{l}
x_{l} \\
y_{l}
\end{array}\right)+\left(\begin{array}{cc}
\cos \theta_{l} & -\sin \theta_{l} \\
\sin \theta_{l} & \cos \theta_{l}
\end{array}\right)\left(\begin{array}{l}
x_{d} \\
y_{d}
\end{array}\right)
$$

$\left(x_{d}, y_{d}\right)$ are considered as constants, and differentiating both side of (6) will refer to

$$
\left(\begin{array}{c}
\dot{x}_{c} \\
\dot{y}_{c}
\end{array}\right)=\left(\begin{array}{cc}
\cos \theta_{l} & -\sin \theta_{l} \\
\sin \theta_{l} & \cos \theta_{l}
\end{array}\right)\left(\begin{array}{c}
v_{l}-y_{d} \omega_{l} \\
x_{d} \omega_{l}
\end{array}\right)
$$

We can define the formation errors as the Eq. (8).

$$
\left(\begin{array}{c}
x_{e} \\
y_{e}
\end{array}\right)=\left(\begin{array}{cc}
\cos \theta_{f} & \sin \theta_{f} \\
-\sin \theta_{f} & \cos \theta_{f}
\end{array}\right)\left(\begin{array}{l}
x_{c}-x_{o f} \\
y_{c}-y_{o f}
\end{array}\right)
$$

Moreover, the angular difference between the leader and the follower is remarked as (9).

$$
\beta=\theta_{l}-\theta_{f}, \quad \dot{\beta}=\omega_{l}-\omega_{f}
$$


Combining the equations from (4) to (7) and differentiating both sides of (8), formation error model can be expressed as

$$
\begin{aligned}
& \dot{x}_{e}=-v_{f}+\omega_{f} y_{e}+\left(v_{l}-y_{d} \omega_{l}\right) \cos \beta-x_{d} \omega_{l} \sin \beta \\
& \dot{y}_{e}=-\left(d+x_{e}\right) \omega_{f}+\left(v_{l}-y_{d} \omega_{l}\right) \sin \beta+x_{d} \omega_{l} \cos \beta
\end{aligned}
$$

The destination of control law design is to find the appropriate control inputs $\left(v_{f}, \omega_{f}\right)$ to meet the desired outcome:

$$
\lim _{t \rightarrow \infty} x_{e} \rightarrow 0, \lim _{t \rightarrow \infty} y_{e} \rightarrow 0,|\beta|<\sigma(\sigma>0)
$$

\section{Sliding-Mode Controller Design}

\subsection{Sliding surface design}

The most significant process of sliding mode control is to choose the suitable sliding surface. Here, we design the sliding surface which ensures that the formation error will converge to zero when the system trajectory lies on the sliding surface (12).

$$
s=\left[\begin{array}{l}
s_{1} \\
s_{2}
\end{array}\right]=\left[\begin{array}{c}
x_{e} \cos \beta+y_{e} \sin \beta \\
-x_{e} \sin \beta+y_{e} \cos \beta
\end{array}\right]
$$

Let $V=0.5 s^{T} s$, thus we can deduce $V=0.5\left(s_{1}^{2}+s_{2}^{2}\right)$ $=0.5\left(x_{e}^{2}+y_{e}^{2}\right)$ from equation (12), which indicates that if $s_{1} \rightarrow 0, s_{2} \rightarrow 0$, the $x_{e} \rightarrow 0, y_{e} \rightarrow 0$ will hold. Hence, when the sliding surface converges to zero, the geometric shapes of the robot formation will be established.

Differentiating the formula (12) will conclude (13)

$$
\begin{aligned}
& \dot{s}_{1}=-v_{f} \cos \beta-d \omega_{f} \sin \beta+v_{l}-y_{d} \omega_{l}+s_{2} \omega_{l} \\
& \dot{s}_{2}=v_{f} \sin \beta-d \omega_{f} \cos \beta+x_{d} \omega_{l}-s_{1} \omega_{l}
\end{aligned}
$$

Furthermore, the formation controller can be simply written as:

$$
\begin{aligned}
& v_{f}=\left(v_{l}-y_{d} \omega_{l}\right) \cos \beta-x_{d} \omega_{l} \sin \beta+y_{e} \omega_{l}-\dot{s}_{1} \cos \beta+\dot{s}_{2} \sin \beta \\
& \omega_{f}=\left[\left(v_{l}-y_{d} \omega_{l}\right) \sin \beta+x_{d} \omega_{l} \cos \beta-x_{e} \omega_{l}-\dot{s}_{1} \sin \beta-\dot{s}_{2} \cos \beta\right] / d
\end{aligned}
$$

\subsection{Controller design with the reaching law}

In this paper, a continuous reaching law is adopted to make each state reaching the sliding surface. Comparing with the switching function of general reaching law, a saturation function is taken in order to reduce the chattering which caused by the computation time delays and limitations of control. The reaching law is as follow:

$$
\dot{s}=-\varepsilon s-k f(s)
$$

Here, $f(s)$ is given as $(16)$ and $\operatorname{sgn}(s)$ is a sign function Besides, $\varepsilon=\left(\varepsilon_{1}, \varepsilon_{2}\right)^{T}, k=\left(k_{1}, k_{2}\right)^{T}, \alpha=\left(\alpha_{1}, \alpha_{2}\right)^{T}, \varphi>0$ and $\varepsilon_{1}, \varepsilon_{2}, k_{1}, k_{2}>0,0<\alpha_{1}, \alpha_{2}<1$.

$$
f_{i}(s)= \begin{cases}\left|s_{i}\right|^{\alpha_{i}} \operatorname{sgn}\left(s_{i}\right) & \text { if }\left|s_{i}\right| \leq \varphi \\ s_{i} \varphi^{\alpha_{i}} /\left|s_{i}\right| & \text { if }\left|s_{i}\right|>\varphi\end{cases}
$$

Thus, we can get the sliding mode controller (17) from equation (12), (14) and (15).

$$
\begin{aligned}
v_{f}= & \left(v_{l}-y_{d} \omega_{l}\right) \cos \beta-x_{d} \omega_{l} \sin \beta+y_{e} \omega_{l}+ \\
& \left(\varepsilon_{1} S_{1}+k_{1} f_{1}(x)\right) \cos \beta-\left(\varepsilon_{2} s_{2}+k_{2} f_{2}(x)\right) \sin \beta \\
\omega_{f}= & \frac{1}{d}\left[\left(v_{l}-y_{d} \omega_{l}\right) \sin \beta+x_{d} \omega_{l} \cos \beta-x_{e} \omega_{l}+\right. \\
& \left.\left(\varepsilon_{1} S_{1}+k_{1} f_{1}(x)\right) \sin \beta+\left(\varepsilon_{2} s_{2}+k_{2} f_{2}(x)\right) \cos \beta\right]
\end{aligned}
$$

Theorem 1. Consider the formation error system (10). Using the control law (17), the sliding surface (12) will converge to zero in finite time $T_{r}$, and $x_{e} \rightarrow 0, y_{e} \rightarrow 0$ as $t=T_{r}$.

Proof. Choose the Lyapunov function as $V_{i}=s_{i}^{2} / 2$ $(i=1,2)$. It is easy to refer to

$$
\dot{V}_{i}=s_{i} \dot{s}_{i}=-\varepsilon_{i} s_{i}^{2}-k_{i} f_{i}\left(s_{i}\right) s_{i}
$$

Case1: if $\left|s_{i}\right|<\varphi$, the equation (18) will be converted to $\dot{V}_{i}=-2 \varepsilon_{i} V_{i}-2^{\left(1+\alpha_{i}\right) / 2} k_{i} V_{i}^{\left(1+\alpha_{i}\right) / 2}$. Define $V=V_{i}(0)$ at $t=0$. By solving differential equation, we can deduce formula

$$
V_{i}^{\frac{1-\alpha_{i}}{2}}=-2^{\frac{\alpha_{i}-1}{2}} \frac{k}{\varepsilon}+\left(2^{\frac{\alpha_{i}-1}{2}} \frac{k}{\varepsilon}+V_{i}^{\frac{1-\alpha_{i}}{2}}(0)\right) e^{-\left(1-\alpha_{i}\right) \varepsilon t}
$$

Let $V_{i}=0$; the reaching time can be expressed as

$$
T_{r i}=\frac{1}{\left(1-\alpha_{i}\right) \varepsilon} \ln \left(\frac{2^{\frac{\alpha_{i}-1}{2}} \frac{k}{\varepsilon}+V_{i}^{\frac{1-\alpha_{i}}{2}}(0)}{2^{\frac{\alpha_{i}-1}{2}} k}\right)
$$

Therefore, $s_{i}(i=1,2)$ will converge to zero at $t=T_{r}=$ $\max \left(T_{r i}\right)(i=1,2)$ and $x_{e} \rightarrow 0, y_{e} \rightarrow 0$ as $t=T_{r}$.

Case 2: if $\left|s_{i}\right|>\varphi$, the equation (18) can be presented as the formula (21).

$$
\dot{V}_{i}=-2 \varepsilon_{i} s_{i}^{2}-k_{i}\left|s_{i}\right| \varphi^{\alpha_{i}}<0
$$

Consequently, there must be a $T_{r \varphi}$ which makes $s_{i} \rightarrow \varphi$. Thus, formation error model will be globally finite time stable. 
Theorem 2. Consider the equation (9) with the control law (17). $\beta$ will be bounded, if the velocity of the leader $0<v_{l}<$ const, $\left|\beta_{0}\right|<\pi$ at $t=0$ and the boundedness of the angular velocity $\omega_{l}$ hold.

Proof. Combining the formula (9) with the control law, we can obtain the equation (22).

$$
\begin{aligned}
& \dot{\beta}=-\left(v_{l} \sin \beta\right) / d+\phi(t) \\
& \phi(t)=\omega_{l}-\frac{1}{d}\left[-y_{d} \omega_{l} \sin \beta+x_{d} \omega_{l} \cos \beta-x_{e} \omega_{l}+\right. \\
& \left.\left(\varepsilon_{1} s_{1}+k_{1} f_{1}\left(s_{1}\right)\right) \cos \beta+\left(\varepsilon_{2} s_{2}+k_{2} f_{2}\left(s_{2}\right)^{\alpha_{2}}\right) \sin \beta\right]
\end{aligned}
$$

The nominal system can be given as $\dot{\beta}=-\left(v_{l} \sin \beta\right) / d$ at $\phi=0$. Select the Lyapunov function as $V_{\beta}=1-\cos \beta$. So we can get $\dot{V}_{\beta}=-\left(v_{l} \sin ^{2} \beta\right) / d \leq 0$ and $V_{\beta}$ is nonincreasing. $\left|\beta_{0}\right|<\pi$ at $t=0$, there must exist a positive constant $\delta$ which meets $\beta_{0} \in[-\pi+\delta, \pi-\delta]$. Then, $V_{\beta}$ $\in[0,2-\delta), \beta(t) \in[-\pi+\delta, \pi-\delta]$ and $\dot{V}_{\beta} \leq-\left(v_{l} / d\right) \delta V_{\beta}$. Besides, $V_{\beta}=2 \sin ^{2}(\beta / 2)$, it is easy to obtain $2 \beta^{2} / \pi^{2}$ $<V_{\beta}<\beta^{2} / 2$ at $\beta \in(-\pi, \pi)$. Thus, the nominal system is exponentially stable using the Lyapunov method. Obviously, $\omega_{l}, x_{e}, s_{1}, s_{2}, \sin \beta$ and $\cos \beta$ are bounded, which can be used to deduce the conclusion that $\phi(t)$ is bounded. Therefore, in the system (21), $\beta$ is bounded by using the stability theory of perturbed systems.

\section{Simulation Results}
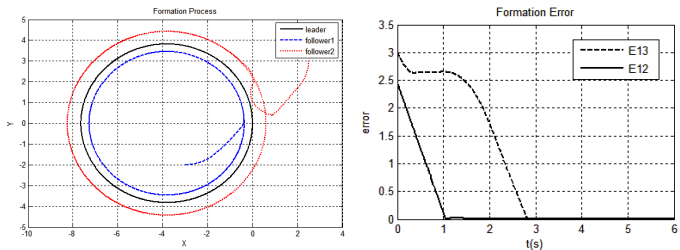

Fig. 3. Formation process and formation errors.
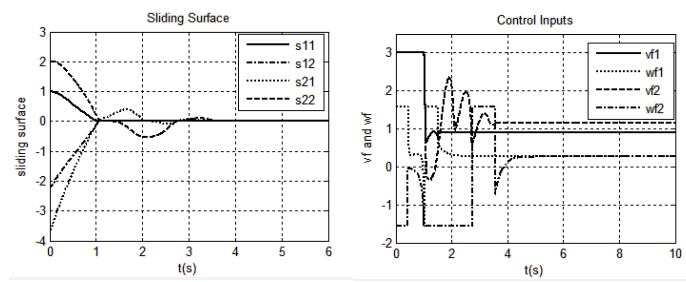

Fig. 4. The sliding surface and inputs of followers.

Three robots are expected to keep a triangle formation and the expected formation shapes are designed as $x_{d 1}=$ $x_{d 2}=-1 \mathrm{~m}, y_{d 1}=0.5 \mathrm{~m}, y_{d 3}=-0.5 \mathrm{~m}$. The movement of the leader robot is set as the circular motion with $v_{l}=1 \mathrm{~m} / \mathrm{s}, \omega_{l}=\pi / 12 \mathrm{rad} / \mathrm{s}$, and the initial poses of three robots are $(0,0, \pi / 2),(-3,-2,0)$ and $(2.5,-3$, $-\pi / 2)$. Moreover, Controller (16) is used with $\varepsilon_{1}=\varepsilon_{2}=5$,

$k_{1}=k_{2}=7, \alpha_{1}=\alpha_{2}=1 / 3, \varphi=5, d_{2}=d_{3}=0.25 \mathrm{~m}$. The control inputs are limited as $v_{f} \leq 3 \mathrm{~m} / \mathrm{s}, \omega_{f} \leq \pi / 2 \mathrm{rad} / \mathrm{s}$.

The formation error is denoted as $E=\sqrt{x_{e}^{2}+y_{e}^{2}}$, The simulation results are presented in Fig. 3 and Fig.4.

\section{Conclusion}

In this paper, the formation control problem of the nonholonomic mobile robot has been solved. A controller with sliding mode is proposed using a continuous reaching law. The simulation results show that the control law can guarantee great formation performance and the specified geometrical shapes of multi-mobile robots will be achieved in finite time.

\section{Acknowledgements}

This work was supported by the NSFC (61327807, 61521091, 61520106010, 61134005) and the National Basic Research Program of China (973 Program: 2012CB821200, 2012CB821201).

\section{References}

1. J. P. Desai, J. P. Ostrowski, and V. Kumar, "Modeling and control of formations of nonholonomic mobile robots," IEEE Trans. Robot. Autom, vol. 17, no. 6, pp. 905-908, Dec. 2001.

2. X. Li, J. Xiao, and Z. Cai, "Backstepping based multiple mobile robots formation control," in Proc. Conf. Intell. Robots Syst., 2005, pp. 887-892.

3. Park, Bong Seok, B. P. Jin, and Y. H. Choi. "Robust formation control of electrically driven nonholonomic mobile robots via sliding mode technique." International Journal of Control, Automation and Systems 9.5(2011): 888-894.

4. Peng, Zhaoxia, et al. "Leader-follower formation control of nonholonomic mobile robots based on a bioinspired neurodynamic based approach." Robotics \& Autonomous Systems 61.9(2013):988-996.

5. Chen, X., and Y. Jia. "Input-constrained formation control of differential-drive mobile robots: geometric analysis and optimization." IET Control Theory \& Applications 8.7 (2014):522-533. 